\title{
Neurons on a chip - toward high throughput network and pharmacology investigations
}

\author{
John Michael Nagarah* \\ Broad Fellows Program, Division of Biology, California Institute of Technology, Pasadena, CA, USA \\ ${ }^{*}$ Correspondence: jnagarah@caltech.edu
}

\section{A commentary on}

From understanding cellular function to novel drug discovery: the role of planar patch-clamp array chip technology

by Py, C., Martina, M., Diaz-Quijada, G. A., Luk, C. C., Martinez, D., Denhoff, $M$. W., Charrier, A., Comas, T., Monette, R., Krantis, A., Syed, N. I., and Mealing, G. A. R. (2011). Front. Pharmacol. 2:51. doi: 10.3389/ fphar.2011.00051

The biophysical properties of single neurons and synaptic connections between populations of neurons enable the brain to form memories, make decisions, and perceive the surrounding environment. The ability of neurons to transmit signals is reliant on ion channel proteins embedded in the plasma membrane (Hodgkin and Huxley, 1952; Koch, 2005). Ion channel defects and neural network dysfunction are the basis of several neurological disorders (Ackerman and Clapham, 1997; Palop et al., 2006; Seeley et al., 2009). The development of new technologies, such as those mentioned within this issue, is fueled by the desire not only to understand the fundamental processes underlying neuronal signals, but also, to gain a better understanding of the causes, and thus potential treatments for neurological diseases.

Traditionally, ion channel protein activity in single cells has been monitored using the glass pipette patch-clamp technique (Hamill et al., 1981). Our detailed understanding of ion channel kinetics and pharmacological properties made possible by this technique is one of the reasons why so many drug targets include ion channels (Wang and Li, 2003). However, the patch-clamp technique has been limited by the number of cells from which it can simultaneously record. Recent advances in robotics have enabled measurements in brain slices from 12 simultaneous pipette patch-clamp electrodes, illustrating novel electrophysiological principles that govern network behavior, though these experiments are still demanding on the user (Anastassiou et al., 2011). In contrast, multi-electrode arrays (MEAs) and silicon-based transistor arrays allow for extracellular recordings from hundreds and thousands of electrodes simultaneously (Pine, 1980; Hutzler et al., 2006). Despite the limited amount of biophysical information obtained from these experiments, the high throughput of MEAs has enabled their successful use in discovering novel network mechanisms (Hosoya et al., 2005) and in screening potential pharmaceutical compounds (Hill et al., 2010; Redon et al., 2010). As each technique presents its own strengths and weaknesses, combining the throughput of the MEA platform with the resolution and control of the patchclamp would provide a unique hybrid that would be highly welcomed in many areas of neuroscience.

Recently, researchers have succeeded in replacing the glass pipette with a micromachined orifice in a planar substrate. Through their ability to achieve an electrical resistance greater than 1 gigaohm between the cell membrane and pore, planar patch-clamp electrodes have enabled high quality electrophysiology recordings (Fertig et al., 2002; Nagarah et al., 2010). Automated patch-clamp (APC) systems have been used primarily for pharmaceutical screening against ion channels of interest expressed in cell lines. Only recently have APCs been used to investigate primary cells or physiologically more relevant cells derived from pluripotent stem cells (Milligan et al., 2009; Stoelzle et al., 2011). In this issue, Py et al. (2011) describe their success in integrating cultured neurons onto planar patch-clamp electrodes. A significant accomplishment of theirs is obtaining gigaohm seals between poly-Llysine (PLL) coated apertures and cultured neurons. This is surprising consider- ing the contamination susceptibility of patch-clamp electrodes, suggesting the sealing mechanism of suspended cells is different than that of cultured neurons. Their platform has allowed for intracellular recordings of neuronal activity from cultured neurons and synapse formation on the chip, demonstrated by dual planar and pipette measurements along with pharmacological characterization. Simultaneous recordings from synaptically connected neurons with dual planar electrodes have proven difficult so far, but nonetheless, they are able to record from multiple apertures simultaneously using an improved device fabrication scheme and microfluidic channels. The authors are also making advancements in chemical patterning methods to precisely localize neurons onto the planar orifices which will eliminate the need for manual placement.

While the bulk of recordings have been from snail neurons, the authors have recently integrated mammalian neurons onto their device, increasing the applicability of this platform to drug screening. High throughput pharmaceutical screening on patch-clamped neuronal cultures will enable the observation of a drug's effect on synaptic transmission as well as ion channel behavior. Additionally, the planar nature of the devices described here opens up the possibility of integrating with it other technologies. For example, a novel microfluidic culture system has allowed for investigations of the cell biology of synaptic functions with high spatial and temporal resolution (Taylor et al., 2010). Combining a similar system with planar patch-clamp electrodes would add electrophysiological control to cell biology experiments, and provide even more detail to synaptic dynamics investigations. Further development is still needed before such experiments are made possible, but Py et. al.'s efforts will undoubtedly contribute to the growing list 
of tools that neuroscientists use to provide insight in neural computation, the nature of neurological diseases, and the treatment of diseases.

\section{REFERENCES}

Ackerman, M. J., and Clapham, D. E. (1997). Ion channels - basic science and clinical disease. N. Engl. J. Med. 336, 1575-1586.

Anastassiou, C. A., Perin, R., Markram, H., and Koch, C. (2011). Ephaptic coupling of cortical neurons. Nat. Neurosci. 14, 217-223.

Fertig, N., Klau, M., George, M., Blick, R. H., and Behrends, J. C. (2002). Activity of single ion channel proteins detected with a planar microstructure. Appl. Phys. Lett. 81, 4865-4867.

Hamill, O. P., Marty, A., Neher, E., Sakmann, B., and Sigworth, F. J. (1981). Improved patch-clamp techniques for high-resolution current recording from cells and cell-free membrane patches. Pflugers Arch. 391, 85-100.

Hill,A. J., Jones, N. A., Williams, C. M., Stephens, G. J., and Whalley, B. J. (2010). Development of multi-electrode array screening for anticonvulsants in acute rat brain slices. J. Neurosci. Methods 185, 246-256.

Hodgkin, A. L., and Huxley, A. F. (1952). A quantitative description of membrane current and its application to conduction and excitation in nerve. J. Physiol. (Lond.) 117, 500 .

Hosoya, T., Baccus, S.A., and Meister, M. (2005). Dynamic predictive coding by the retina. Nature 436, 71-77.
Hutzler, M., Lambacher, A., Eversmann, B., Jenkner, M. Thewes, R., and Fromherz, P. (2006). High-resolution multitransistor array recording of electrical field potentials in cultured brain slices. J. Neurophysiol. 96, 1638.

Koch, C. (2005). Biophysics of Computation: Information Processing in Single Neurons. New York, NY: Oxford University Press.

Milligan, C. J., Li, J., Sukumar, P., Majeed, Y., Dallas, M. L., English, A., Emery, P., Porter, K. E., Smith, A. M., and Mcfadzean, I. (2009). Robotic multi-well planar patch-clamp for native and primary mammalian cells. Nat. Protoc. 4, 244

Nagarah, J. M., Paek, E., Luo, Y., Wang, P., Hwang, G. S., and Heath, J. R. (2010). Batch fabrication of high performance planar patch clamp devices in quartz. Adv. Mater. 22, 4622-4627.

Palop, J. J., Chin, J., and Mucke, L. (2006). A network dysfunction perspective on neurodegenerative diseases. Nature 443, 768

Pine, J. (1980). Recording action potentials from cultured neurons with extracellular microcircuit electrodes. $J$. Neurosci. Methods 2, 19-31.

Py, C., Martina, M., Diaz-Quijada, G. A., Luk, C. C. Martinez, D., Denhoff, M. W., Charrier, A., Comas, T., Monette, R., Krantis, A., Syed, N. I., and Mealing, G. A. R. (2011). From understanding cellular function to novel drug discovery: the role of planar patch-clamp array chip technology. Front. Pharmacol. 2:51. doi: 10.3389/fphar.2011.00051

Redon, N., Lanneau, C., Cervello, P., Biton, B., Daniel, B. Oury-Donat, F., and Avenet, P. (2010). "Importanice of multielectrode array recordings in drug discov- ery," in Seventh International Meeting on SubstrateIntegrated Microelectrode Arrays, ed.A. Stett (BIOPRO Baden-Wurttemberg GmbH), Stuttgart, 167-168.

Seeley, W. W., Crawford, R. K., Zhou, J., Miller, B. L., and Greicius, M.D. (2009).Neurodegenerative diseases target large-scale human brain networks. Neuron 62, 42-52.

Stoelzle, S., Haythornthwaite, A., Kettenhofen, R., Kolossov, E., Bohlen, H., George, M., Brüggemann, A., and Fertig, N. (2011). Automated patch clamp on meSc-derived cardiomyocytes for cardiotoxicity prediction. J. Biomol. Screen. 16, 910-916.

Taylor, A. M., Dieterich, D. C., Ito, H. T., Kim, S. A., and Schuman, E. M. (2010). Microfluidic local perfusion chambers for the visualization and manipulation of synapses. Neuron 66, 57-68.

Wang, X., and Li, M. (2003). Automated electrophysiology: high throughput of art. Assay Drug Dev. Technol. 1, 695-708.

Received: 10 October 2011; accepted: 06 November 2011; published online: 23 November 2011.

Citation: Nagarah JM (2011) Neurons on a chip - toward high throughput network and pharmacology investigations. Front. Pharmacol. 2:74. doi: 10.3389/fphar.2011.00074 This article was submitted to Frontiers in Pharmacology of Ion Channels and Channelopathies, a specialty of Frontiers in Pharmacology.

Copyright (C) 2011 Nagarah. This is an open-access article subject to a non-exclusive license between the authors and Frontiers Media SA, which permits use, distribution and reproduction in other forums, provided the original authors and source are credited and other Frontiers conditions are complied with. 